\title{
Kink and fluting modes of stratified coronal magnetic loops with elliptical cross-sections
}

\author{
R. J. Morton and M. S. Ruderman
}

Solar Physics and Space Plasma Research Centre ( $\left.\mathrm{SP}^{2} \mathrm{RC}\right)$, University of Sheffield, Hicks Building, Hounsfield Road, Sheffield S3 7RH, UK

e-mail: [r.j.morton;m.s.ruderman]@sheffield.ac.uk

Received 29 October 2010 / Accepted 20 December 2010

\section{ABSTRACT}

\begin{abstract}
Aims. We study kink and fluting oscillations of a straight magnetic tube with an elliptic cross-section and density varying along the tube.

Methods. The governing equations for kink and fluting modes in the thin tube approximation are derived. We found that there are two kink modes, polarised along the semimajor and semiminor axes of the elliptic cross-section. We have shown that the ratio of frequencies of the first overtone and fundamental mode is the same for both kink modes and independent of the ratio of the ellipse axes.

Results. On the basis of this result we concluded that the estimates of the atmospheric scale height obtained using simultaneous observations of the fundamental mode and first overtone of the coronal loop kink oscillations are independent of the ellipticity of the loop cross-section.
\end{abstract}

Key words. magnetohydrodynamics (MHD) - plasmas - Sun: corona - Sun: oscillations - waves

\section{Introduction}

The solar atmosphere is a highly dynamic and structured plasma that is able to support a wide variety of magneto-acoustic waves and oscillations. Each layer of the solar atmosphere, from the photosphere to the corona, is magnetically connected to the others via the all pervading magnetic field. The omnipresence of the waves throughout the atmosphere is becoming well documented as new and exciting techniques are being developed to help observe and study the waves (see, e.g. Banerjee et al. 2007; Tomczyk et al. 2007).

After transverse coronal loop oscillations were first observed by TRACE in 1998 (Aschwanden et al. 1999; Nakariakov et al. 1999), the phenomenon became one of the hot topics within solar physics. In the first theoretical interpretation of these oscillations, a coronal loop was modelled as a straight magnetic cylinder with the density constant inside and outside. Since then, a number of more complicated and realistic models have been considered. For a recent review on the theory of transverse oscillations of a coronal loop see, e.g., Ruderman \& Erdélyi (2009).

Although the transverse coronal loop oscillations are interesting on their own, their main importance is related to the fact that they are a powerful tool of coronal seismology. Nakariakov \& Ofman (2001) demonstrated this by using the observations of transverse coronal loop oscillations to estimate the magnitude of the magnetic field in the corona, while Andries et al. (2005) suggested to use these observations to estimate the atmospheric scale height in the corona.

In this paper we continue to study the transverse oscillations of coronal loops. Coronal loops with elliptical cross-sections and a constant density profile have been studied previously in both cold (Ruderman 2003) and finite- $\beta$ (Erdélyi \& Morton 2009) plasmas. Now, we consider oscillations of loops with the density varying along the loop and a constant elliptic cross-section. The paper is organized as follows. In the next section we formulate the problem. In Sect. 3 we derive the governing equations for kink and fluting oscillations of a coronal loop with an elliptic cross-section in the thin tube approximation. In Sect. 4 we study the implication of our analysis on coronal seismology. Section 5 contains the summary of the obtained results and our conclusions.

\section{Problem formulation}

We model a coronal loop as a straight magnetic tube with an elliptical cross-section. The cold plasma approximation is used. The density varies along the tube, while the cross-section remains constant. In Cartesian coordinates $x, y, z$ the loop axis coincides with the $z$-axis. The equilibrium magnetic field is given by $\boldsymbol{B}=B \hat{z}$, where $B$ is constant and $\hat{z}$ is the unit vector in the $z$-direction. The plasma motion is governed by the linearised ideal MHD equations,

$$
\begin{aligned}
& \frac{\partial^{2} \boldsymbol{\xi}}{\partial t^{2}}=\frac{1}{\mu_{0} \rho}(\nabla \times \boldsymbol{b}) \times \boldsymbol{B}, \\
& \boldsymbol{b}=\nabla \times(\boldsymbol{\xi} \times \boldsymbol{B}) .
\end{aligned}
$$

Here $\boldsymbol{\xi}$ is the plasma displacement, $\boldsymbol{b}$ the magnetic field perturbation, $\rho(z)$ the equilibrium density, and $\mu_{0}$ the magnetic permeability of free space; $\rho(z)=\rho_{\mathrm{i}}(z)$ inside the tube and $\rho(z)=\rho_{\mathrm{e}}(z)$ outside the tube.

Let us introduce the elliptic coordinates $s$ and $\varphi$ in the $x y$-plane (see Fig. 1). The Cartesian coordinates are expressed in terms of elliptic coordinates as

$$
x=\sigma \cosh s \cos \varphi, \quad y=\sigma \sinh s \sin \varphi,
$$

where $\sigma$ is a quantity with the dimension of length, $s$ varies from 0 to $\infty$, and $\varphi$ from $-\pi$ to $\pi$. In the elliptic coordinates the equation of the tube boundary is $s=s_{0}$. Then the semimajor 


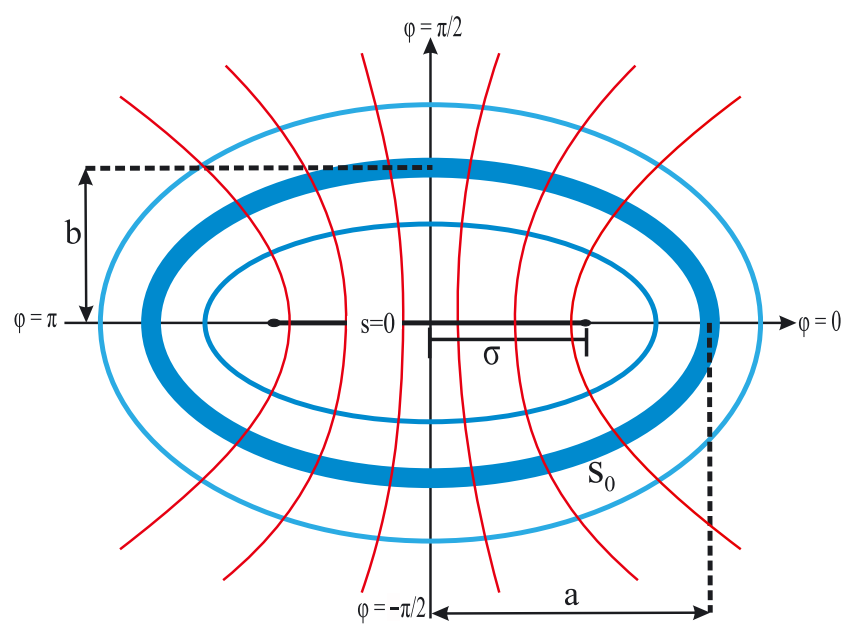

Fig. 1. Sketch showing the elliptical coordinate system used to describe the loop cross-section. The open and closed curves show the $s$ and $\varphi$ coordinate lines respectively. The thick closed curve shows the tube boundary.

and semiminor half-axes of the tube elliptic cross-section are in the $x$ and $y$-direction, and they are given by

$$
a=\sigma \cosh s_{0}, \quad b=\sigma \sinh s_{0} .
$$

At the tube boundary the normal component of the displacement, $\xi_{s}$, and the magnetic pressure perturbation, $P=\boldsymbol{b} \cdot \boldsymbol{B} / \mu_{0}$, has to be continuous,

$$
\llbracket \xi_{s} \rrbracket=0, \quad \llbracket P \rrbracket=0 \quad \text { at } \quad s=s_{0},
$$

where $\llbracket f \rrbracket$ indicates the jumps of function $f$ across the boundary defined as

$$
\llbracket f \rrbracket=\lim _{\varepsilon \rightarrow 0}[f(s+\varepsilon)-f(s-\varepsilon)] .
$$

The magnetic field lines at the loop foot points are frozen in the dense photospheric plasma, so that

$$
\boldsymbol{\xi}=0 \quad \text { at } \quad z= \pm L / 2,
$$

where $L$ is the loop length.

It follows from Eq. (3) that the points with the elliptical coordinates $s=0, \varphi=\varphi_{0}$, and $s=0, \varphi=-\varphi_{0}$ are the same point in the $x y$-plane. This implies that $P$ and $\xi_{s}$ have to satisfy the boundary conditions

$$
P(0, \varphi)=P(0,-\varphi), \quad \xi_{s}(0, \varphi)=-\xi_{s}(0,-\varphi) .
$$

Equations (1) and (2) together with the boundary conditions (5), (7) and (8) will be used in the next section to derive the governing equations for kink and fluting oscillations in the thin tube approximation.

\section{Derivation of governing equations}

The analysis in this section is similar to one used by Dymova \& Ruderman (2005) to derive the governing equation for a thin tube with a circular tube cross-section. We begin by noting that, in accordance with Eq. (1), $\xi_{z}=0$. The system of Eqs. (1) and (2) can then be transformed to

$$
\begin{aligned}
\frac{\partial^{2} \boldsymbol{\xi}}{\partial t^{2}} & =-\frac{1}{\rho} \nabla_{\perp} P+\frac{B}{\mu_{0} \rho} \frac{\partial \boldsymbol{b}_{\perp}}{\partial z}, \\
\boldsymbol{b}_{\perp} & =B \frac{\partial \boldsymbol{\xi}}{\partial z} \\
P & =-\rho v_{\mathrm{A}}^{2} \nabla \cdot \boldsymbol{\xi},
\end{aligned}
$$

where $v_{\mathrm{A}}$ is the Alfvén speed defined by $v_{\mathrm{A}}^{2}=B^{2} / \mu_{0} \rho$, and the operator $\nabla_{\perp}$ and component of the magnetic field perturbation perpendicular to the $z$-axis are given by

$$
\nabla_{\perp}=\nabla-\hat{z} \frac{\partial}{\partial z}, \quad \boldsymbol{b}_{\perp}=\boldsymbol{b}-\boldsymbol{b} \cdot \hat{z} .
$$

Eliminating $\boldsymbol{b}_{\perp}$ from Eqs. (9) and (10) yields

$$
\frac{\partial^{2} \boldsymbol{\xi}}{\partial t^{2}}-v_{\mathrm{A}}^{2} \frac{\partial^{2} \boldsymbol{\xi}}{\partial z^{2}}=-\frac{1}{\rho} \nabla_{\perp} P .
$$

Taking the divergence of this equation and using Eq. (11) we arrive at the equation for $P$,

$$
\frac{\partial^{2} P}{\partial t^{2}}-v_{\mathrm{A}}^{2} \frac{\partial^{2} P}{\partial z^{2}}=v_{\mathrm{A}}^{2} \nabla_{\perp}^{2} P .
$$

Now we use the thin tube approximation. To do this we note that the characteristic spatial scale in the $z$-direction is $L$, and the characteristic time of the problem is $L / \bar{v}_{\mathrm{A}}$, where $\bar{v}_{\mathrm{A}}$ is a typical value of Alfvén speed. In what follows we only consider the perturbations that decay at the distance of a few $a$ from the tube. Then the characteristic spatial scale in the $x$ and $y$-direction is $a$. It follows from this analysis that the ratio of the left-hand side of Eq. (14) to its right-hand side is of the order of $(a / L)^{2} \ll 1$, so that we can neglect the left-hand side. Then, using the expression for $\nabla_{\perp}^{2}$ in the elliptical coordinates (e.g. Korn \& Korn 1961), we obtain the equation for $P$ in the thin tube approximation,

$$
\frac{\partial^{2} P}{\partial s^{2}}+\frac{\partial^{2} P}{\partial \varphi^{2}}=0
$$

The solution to this equation has to satisfy the first regularity condition in Eq. (8), and the second boundary condition in Eq. (5). Using Eq. (13) we rewrite the second regularity condition in terms of $P$,

$$
\left.\frac{\partial P(s, \varphi)}{\partial s}\right|_{s=0}=-\left.\frac{\partial P(s,-\varphi)}{\partial s}\right|_{s=0} .
$$

To derive the governing equations for non-axisymmetric tube oscillations we solve Eqs. (13) and (15) inside and outside the tube, and then match the two solutions at the tube boundary. It is straightforward to obtain the general solution to Eq. (15) inside the tube satisfying the regularity conditions Eqs. (8) and (16),

$$
P^{\mathrm{i}}=\sum_{n=1}^{\infty}\left[C_{n}^{\mathrm{i}} \cosh (n s) \cos (n \varphi)+D_{n}^{\mathrm{i}} \sinh (n s) \sin (n \varphi)\right],
$$

where $C_{n}^{\mathrm{i}}$ and $D_{n}^{\mathrm{i}}$ are arbitrary functions of $t$ and $z$. The solution outside the tube has to decay as $s \rightarrow \infty$. Hence, its general form is

$$
P^{\mathrm{e}}=\sum_{n=1}^{\infty} \mathrm{e}^{-n s}\left[C_{n}^{\mathrm{e}} \cos (n \varphi)+D_{n}^{\mathrm{e}} \sin (n \varphi)\right]
$$

where once again $C_{n}^{\mathrm{e}}$ and $D_{n}^{\mathrm{e}}$ are arbitrary functions of $t$ and $z$. Substituting Eqs. (17) and (18) in Eq. (13) and using the expression for $\nabla_{\perp}$ in the elliptical coordinates (e.g. Korn \& Korn 1961),

$$
\nabla_{\perp}=\frac{1}{\sigma \Theta}\left(\hat{s} \frac{\partial}{\partial s}+\hat{\varphi} \frac{\partial}{\partial \varphi}\right), \quad \Theta=\left(\sinh ^{2} s+\sin ^{2} \varphi\right)^{1 / 2},
$$

where $\hat{s}$ and $\hat{\varphi}$ are the unit vectors in the $s$ and $\varphi$-direction, we obtain the expressions for $\xi_{s}$ inside and outside the tube,

$$
\xi_{s}^{\mathrm{i}}=\frac{1}{\sigma \Theta} \sum_{n=1}^{\infty}\left[F_{n}^{\mathrm{i}} \sinh (n s) \cos (n \varphi)+G_{n}^{\mathrm{i}} \cosh (n s) \sin (n \varphi)\right],
$$




$$
\xi_{s}^{\mathrm{e}}=\frac{1}{\sigma \Theta} \sum_{n=1}^{\infty} \mathrm{e}^{-n s}\left[F_{n}^{\mathrm{e}} \cos (n \varphi)+G_{n}^{\mathrm{e}} \sin (n \varphi)\right] .
$$

In these equations $F_{n}^{\mathrm{i}}, G_{n}^{\mathrm{i}}, F_{n}^{\mathrm{e}}$ and $G_{n}^{\mathrm{e}}$ are functions of $t$ and $z$. They are related to the functions $C_{n}^{\mathrm{i}} D_{n}^{\mathrm{i}}, C_{n}^{\mathrm{e}}$ and $D_{n}^{\mathrm{e}}$ by

$$
\begin{aligned}
& \frac{\partial^{2} F_{n}^{\mathrm{i}}}{\partial t^{2}}-v_{\mathrm{Ai}}^{2} \frac{\partial^{2} F_{n}^{\mathrm{i}}}{\partial z^{2}}=-\frac{C_{n}^{\mathrm{i}}}{\rho_{\mathrm{i}}} \\
& \frac{\partial^{2} G_{n}^{\mathrm{i}}}{\partial t^{2}}-v_{\mathrm{Ai}}^{2} \frac{\partial^{2} G_{n}^{\mathrm{i}}}{\partial z^{2}}=-\frac{D_{n}^{\mathrm{i}}}{\rho_{\mathrm{i}}} \\
& \frac{\partial^{2} F_{n}^{\mathrm{e}}}{\partial t^{2}}-v_{\mathrm{Ae}}^{2} \frac{\partial^{2} F_{n}^{\mathrm{e}}}{\partial z^{2}}=\frac{C_{n}^{\mathrm{e}}}{\rho_{\mathrm{e}}} \\
& \frac{\partial^{2} G_{n}^{\mathrm{e}}}{\partial t^{2}}-v_{\mathrm{Ae}}^{2} \frac{\partial^{2} G_{n}^{\mathrm{e}}}{\partial z^{2}}=\frac{D_{n}^{\mathrm{e}}}{\rho_{\mathrm{e}}}
\end{aligned}
$$

Substituting Eqs. (17) and (18) in the second boundary condition in Eq. (5) we obtain

$$
C_{n}^{\mathrm{i}} \cosh \left(n s_{0}\right)=\mathrm{e}^{-n s_{0}} C_{n}^{\mathrm{e}}, \quad D_{n}^{\mathrm{i}} \sinh (n s)=\mathrm{e}^{-n s_{0}} D_{n}^{\mathrm{e}} .
$$

Substituting Eqs. (20) and (21) in the first boundary condition in Eq. (5) yields

$$
F_{n}^{\mathrm{i}} \sinh \left(n s_{0}\right)=\mathrm{e}^{-n s_{0}} F_{n}^{\mathrm{e}}, \quad G_{n}^{\mathrm{i}} \cosh (n s)=\mathrm{e}^{-n s_{0}} G_{n}^{\mathrm{e}} .
$$

Eliminating $C_{n}^{\mathrm{i}}, C_{n}^{\mathrm{e}}$ and $F_{n}^{\mathrm{e}}$ from Eqs. (22), (24), (26) and (27) we obtain the equation for $F_{n}^{\mathrm{i}}$,

$$
\frac{\partial^{2} F_{n}}{\partial t^{2}}-c_{n \mathrm{c}}^{2} \frac{\partial^{2} F_{n}}{\partial z^{2}}=0, \quad c_{n \mathrm{c}}^{2}=\frac{B^{2}\left[1+\tanh \left(n s_{0}\right)\right]}{\mu_{0}\left[\rho_{\mathrm{i}}+\rho_{\mathrm{e}} \tanh \left(n s_{0}\right)\right]},
$$

where we have dropped the superscript "ii". Eliminating $D_{n}^{\mathrm{i}}, D_{n}^{\mathrm{e}}$ and $G_{n}^{\mathrm{e}}$ from Eqs. (23), (25), (26) and (27) we obtain the equation for $G_{n}^{\mathrm{i}}$,

$$
\frac{\partial^{2} G_{n}}{\partial t^{2}}-c_{n \mathrm{~s}}^{2} \frac{\partial^{2} G_{n}}{\partial z^{2}}=0, \quad c_{n \mathrm{~s}}^{2}=\frac{B^{2}\left[1+\tanh \left(n s_{0}\right)\right]}{\mu_{0}\left[\rho_{\mathrm{i}} \tanh \left(n s_{0}\right)+\rho_{\mathrm{e}}\right]},
$$

where we have once again dropped the superscript "i". It follows from Eqs. (7) and (20) that $F_{n}$ and $G_{n}$ have to satisfy the boundary conditions

$$
F_{n}=0, \quad G_{n}=0 \quad \text { at } \quad z= \pm L / 2 .
$$

In Eqs. (28) and (29) $n=1$ corresponds to kink modes, and $n>1$ to fluting modes.

In the elliptical coordinates the loop axis $(x=y=0)$ is defined by $s=0$ and $\varphi=\pi / 2$. It follows from Eq. (20) that the kink mode described by Eq. (28) does not displace the loop axis in the $s$-direction which, at the loop axis, coincides with the $y$ direction. Hence, the loop axis displacement is in the $x$-direction, i.e. this mode is polarised in the direction of the semimajor axis of the tube cross-section. The kink mode described by Eq. (29) displaces the loop axis in the $s$-direction. It is straightforward to show that it does not displace it in the $\varphi$-direction which, at the loop axis, coincides with the $x$-direction. Hence, the loop axis displacement is in the $y$-direction, i.e. this mode is polarised in the direction of the semiminor axis of the tube cross-section.

When the density is constant, we can use Eqs. (28) and (29) with the boundary conditions Eq. (30) to recover the results obtained by Ruderman (2003). Let us look for the eigenmodes and restrict the analysis to the fundamental modes in the $z$ direction. This implies that we take $F_{n}$ and $G_{n}$ proportional to $\mathrm{e}^{-\mathrm{i} \omega t} \cos (\pi z / L)$. Then we immediately obtain that the eigenfrequencies of the boundary value problem defined by Eq. (28) and the boundary conditions (30) are given by

$$
\omega_{n \mathrm{c}}^{2}=\frac{\pi^{2} c_{n \mathrm{c}}^{2}}{L^{2}}=\frac{\pi^{2} B^{2}\left[1+\tanh \left(n s_{0}\right)\right]}{\mu_{0} L^{2}\left[\rho_{\mathrm{i}}+\rho_{\mathrm{e}} \tanh \left(n s_{0}\right)\right]}, \quad n=1,2, \ldots,
$$

and the eigenfrequencies of the boundary value problem defined by Eq. (29) and the boundary conditions (30) are given by

$$
\omega_{n \mathrm{~s}}^{2}=\frac{\pi^{2} c_{n \mathrm{~s}}^{2}}{L^{2}}=\frac{\pi^{2} B^{2}\left[1+\tanh \left(n s_{0}\right)\right]}{\mu_{0} L^{2}\left[\rho_{\mathrm{i}} \tanh \left(n s_{0}\right)+\rho_{\mathrm{e}}\right]} . \quad n=1,2, \ldots
$$

In particular, the squares of eigenfrequencies of the kink modes are given by

$$
\omega_{1 \mathrm{c}}^{2}=\frac{\pi^{2} B^{2}(a+b)}{\mu_{0} L^{2}\left(a \rho_{\mathrm{i}}+b \rho_{\mathrm{e}}\right)}, \quad \omega_{1 \mathrm{~s}}^{2}=\frac{\pi^{2} B^{2}(a+b)}{\mu_{0} L^{2}\left(b \rho_{\mathrm{i}}+a \rho_{\mathrm{e}}\right)} .
$$

It is straightforward to see that the eigenfrequencies satisfy

$$
\omega_{1 \mathrm{c}}<\omega_{2 \mathrm{c}}<\cdots<\omega_{2 \mathrm{~s}}<\omega_{1 \mathrm{~s}}
$$

\section{Implication on coronal seismology}

After Verwichte et al. (2004) reported two cases of observations of the transverse coronal loop oscillations where, in addition to the fundamental mode, the first overtone was also observed, Andries et al. (2005) suggested observations of this nature could be used to estimate the scale height in the solar corona. Andries et al. (2005) assumed that an oscillating loop has a half-circle shape and a circular cross-section, and it is in the vertical plane. They also assumed that the atmosphere is isothermal. In that case, the dependence of the plasma density on $z$ is given by

$$
\rho_{\mathrm{e}}=\rho_{\mathrm{f}} \exp \left(-\frac{L}{\pi H} \cos \frac{\pi z}{L}\right), \quad \rho_{\mathrm{i}}=\zeta \rho_{\mathrm{e}}
$$

where $H$ is the atmospheric scale height, $\rho_{\mathrm{f}}$ the plasma density at the loop foot points outside the loop, and $\zeta>1$ a constant. Andries et al. (2005) calculated the ratio of frequencies of the first overtone and fundamental mode and found that this ratio is a monotonically decreasing function of the parameter $L / H$. Hence, if we know the ratio of frequencies and $L$, we can determine $H$. For a recent review of coronal seismology using kink oscillation overtones see Andries et al. (2009).

A very important question is how robust is this method. Dymova \& Ruderman (2006) and Morton \& Erdélyi (2009a) have found that the account of the loop shape can moderately affect the estimates of the atmospheric scale height. Ruderman (2007) has shown that the twist of magnetic field lines in the loop can be safely neglected when estimating the atmospheric scale height in the corona. Robertson et al. (2010) found that the estimates of the atmospheric scale height obtained using the twothread model are exactly the same as those obtained using the model of a monolithic coronal loop with a circular cross-section of constant radius when the two threads have the same scale height. Recently Ruderman (2010) showed that the account of stationary time independent siphon flows in coronal loops have little influence on the estimates of the coronal scale height found using the frequency ratio. On the other hand, Ruderman et al. (2008) and Verth et al. (2008) found that the account of the loop expansion can strongly affect these estimates. 
In this section we study what the effect the elliptic crosssection has on the estimates of the coronal scale height. As we have already seen, when a loop has an elliptic cross-section, its kink oscillations are polarised along the axes of the crosssection. The kink mode polarised in the direction of the semimajor axis is described by Eq. (28) with $n=1$, while the kink mode polarised in the direction of the semiminor axis is described by Eq. (29) with $n=1$. Let us consider the solutions to these equations in the form of eigenmodes and take $F_{1}$ and $G_{1}$ proportional to $\exp (-\mathrm{i} \omega t)$. Using Eq. (35) we obtain

$$
\begin{aligned}
& c_{1 \mathrm{c}}^{2}=\frac{B^{2}(a+b)}{\mu_{0} \rho_{\mathrm{f}}(a \zeta+b)} \exp \left(\frac{L}{\pi H} \cos \frac{\pi z}{L}\right), \\
& c_{1 \mathrm{~s}}^{2}=\frac{B^{2}(a+b)}{\mu_{0} \rho_{\mathrm{f}}(b \zeta+a)} \exp \left(\frac{L}{\pi H} \cos \frac{\pi z}{L}\right) .
\end{aligned}
$$

Then, introducing

$$
\Omega_{\mathrm{c}}^{2}=\frac{\mu_{0} \rho_{\mathrm{f}}(a \zeta+b) \omega^{2}}{B^{2}(a+b)}, \quad \Omega_{\mathrm{s}}^{2}=\frac{\mu_{0} \rho_{\mathrm{f}}(b \zeta+a) \omega^{2}}{B^{2}(a+b)},
$$

we reduce Eqs. (28) and (29) with $n=1$ to

$$
\frac{d^{2} U}{d z^{2}}+\Omega^{2} U \exp \left(-\frac{L}{\pi H} \cos \frac{\pi z}{L}\right)=0,
$$

where either $U=F_{1}$ and $\Omega=\Omega_{\mathrm{c}}$, or $U=G_{1}$ and $\Omega=\Omega_{\mathrm{s}}$, and $U$ satisfies the boundary conditions $U=0$ at $z= \pm L / 2$. If $a=b$ then we have a circular cross-section and it can be seen from Eqs. (38) that $\Omega_{\mathrm{c}}^{2}=\Omega_{\mathrm{s}}^{2}$. Since Eq. (39) does not contain $a$ and $b$, the equation remains the same for the elliptical crosssection. Hence, the eigenvalues of the boundary value problem for $U$ are independent of $a$ and $b$ and independent of whether the loop has a circular or elliptical cross-section. With respect to observations, the eigenvalue $\Omega$ is related to the observable quantity $\omega$ by a constant scaling factor, where the scaling factor depends upon $a$ and $b$ for the elliptical cross-section. Hence, $\omega_{2} / \omega_{1}=\Omega_{2} / \Omega_{1}$ due to the cancelation of the constant scaling factor. Since

$$
\frac{\Omega_{2 \mathrm{c}}}{\Omega_{1 \mathrm{c}}}=\frac{\Omega_{2}}{\Omega_{1}}, \quad \frac{\Omega_{2 \mathrm{~s}}}{\Omega_{1 \mathrm{~s}}}=\frac{\Omega_{2}}{\Omega_{1}},
$$

it follows that we obtain the same estimates of the atmospheric scale height no matter if we use the observation of the kink oscillations polarised in the direction of the semimajor or semiminor axis. Further, due to the estimates also being independent of $a$ and $b$, they are the same as those obtained for a loop with the circular cross-section.

\section{Summary and conclusions}

In this paper we have studied kink and fluting oscillations of straight magnetic loops with a constant elliptic cross-section and density varying along the loop. We derived the governing equations for kink and fluting modes in the thin tube approximation. All these equations are similar to the equation describing kink oscillations of a straight tube with the circular cross-section. We found that there are two sets of kink and fluting modes, one set polarised in the direction of semimajor axis of the elliptic crosssection, and the other set polarised in the direction of the semiminor axis. The frequencies of fundamental mode and overtones of these two kinds of kink oscillation are different. However, the ratio of frequencies of the first overtone and the fundamental mode is the same for both kink oscillations, and it is independent of the ratio of the ellipse half-axes $a / b$. This result implies that we obtain the same estimates of the atmospheric scale height no matter if we use the observation of the kink oscillations polarised in the direction of semimajor or semiminor axis. The estimates are also the same as those obtained for a loop with the circular cross-section. This demonstrates that the model shows a very robust nature when considering a static plasma. However, if the plasma in the loops is dynamic (i.e. time dependent) then the ability of the static model to provide accurate estimates may become questionable (see e.g. Morton \& Erdélyi 2009b).

It should be noted that the model for coronal loops used here is simplified, with the observed structure of loops appearing to be more complicated. López Fuentes et al. (2006) modelled coronal loops using magnetic extrapolation and observational data. They found that cross-sectional area and shape changed along the axis of the modelled loop. Analytical work by Ruderman (2009), who modelled a curved coronal loop with a potential magnetic field, found that the introduction of curvature caused the cross-section of a coronal loop to change shape, from a circular cross-section at the footpoints to an elliptical crosssection at the apex. Such a model influenced the transverse oscillations of the loop. It was shown by Andries et al. (2005) that the frequency ratio is dependent upon quantities that influence the degree of stratification. In the present discussion, the variable $a$ and $b$ are independent of $z$ so do not influence the frequency ratio. It is possible that the introduction of a variable cross-section and area along a coronal loop could influence the ratio $\omega_{2} / \omega_{1}$.

Acknowledgements. The authors thank the Science and Technology Facilities Council (STFC), UK for the financial support they received. We also thank the referee for a number of useful suggestions.

\section{References}

Andries, J., Arregui, I., \& Goossens, M. 2005, ApJ, 624, L57

Andries, J., van Doorsselaere, T., Roberts, B., et al. 2009, Space Sci. Rev., 149, 3

Aschwanden, M. J., Fletcher, L., Schrijver, C. J., \& Alexander, D. 1999, ApJ, 520,880

Banerjee, D., Erdélyi, R., Oliver, R., \& O’Shea, E. 2007, Sol. Phys., 246, 3

Dymova, M. V., \& Ruderman, M. S. 2005, Sol. Phys., 229, 79

Dymova, M. V., \& Ruderman, M. S. 2006, A\&A, 459, 241

Erdélyi, R., \& Morton, R. J. 2009, A\&A, 494, 295

Korn, G., \& Korn, T. 1961, Mathematical Handbook for Scientists and Engineers (New York: McGraw-Hill)

López Fuentes, M. C., Klimchuk, J. A., \& Démoulin, P. 2006, ApJ, 639, 459

Morton, R., \& Erdélyi, R. 2009a, A\&A, 605, 493

Morton, R. J., \& Erdélyi, R. 2009b, ApJ, 707, 750

Nakariakov, V. M., \& Ofman, L. 2001, A\&A, 372, L53

Nakariakov, V. M., Ofman, L., Deluca, E. E., Roberts, B., \& Davila, J. M. 1999, Science, 285, 862

Robertson, D., Ruderman, M. S., \& Taroyan, Y. 2010, A\&A, 515

Ruderman, M. S. 2003, A\&A, 409, 287

Ruderman, M. S. 2007, Sol. Phys., 246, 119

Ruderman, M. S. 2009, A\&A, 506, 885

Ruderman, M. S. 2010, Sol. Phys., 267, 377

Ruderman, M. S., \& Erdélyi, R. 2009, Space Sci. Rev., 149, 199

Ruderman, M. S., Verth, G., \& Erdélyi, R. 2008, ApJ, 686, 694

Tomczyk, S., McIntosh, S. W., Keil, S. L., et al. 2007, Science, 317, 1192

Verth, G., Erdélyi, R., \& Jess, D. B. 2008, ApJ, 687, L45

Verwichte, E., Nakariakov, V. M., Ofman, L., \& Deluca, E. E. 2004, Sol. Phys., 223, 77 